\title{
FONCTIONNEMENT \\ EN DÉCHARGEUR DES TURBINES DE BASSES CHUTES. \\ ESSAIS SUR MODÈLE RÉDUIT. COMPARAISON \\ AVEC LES RÉSULTATS INDUSTRIELS
}

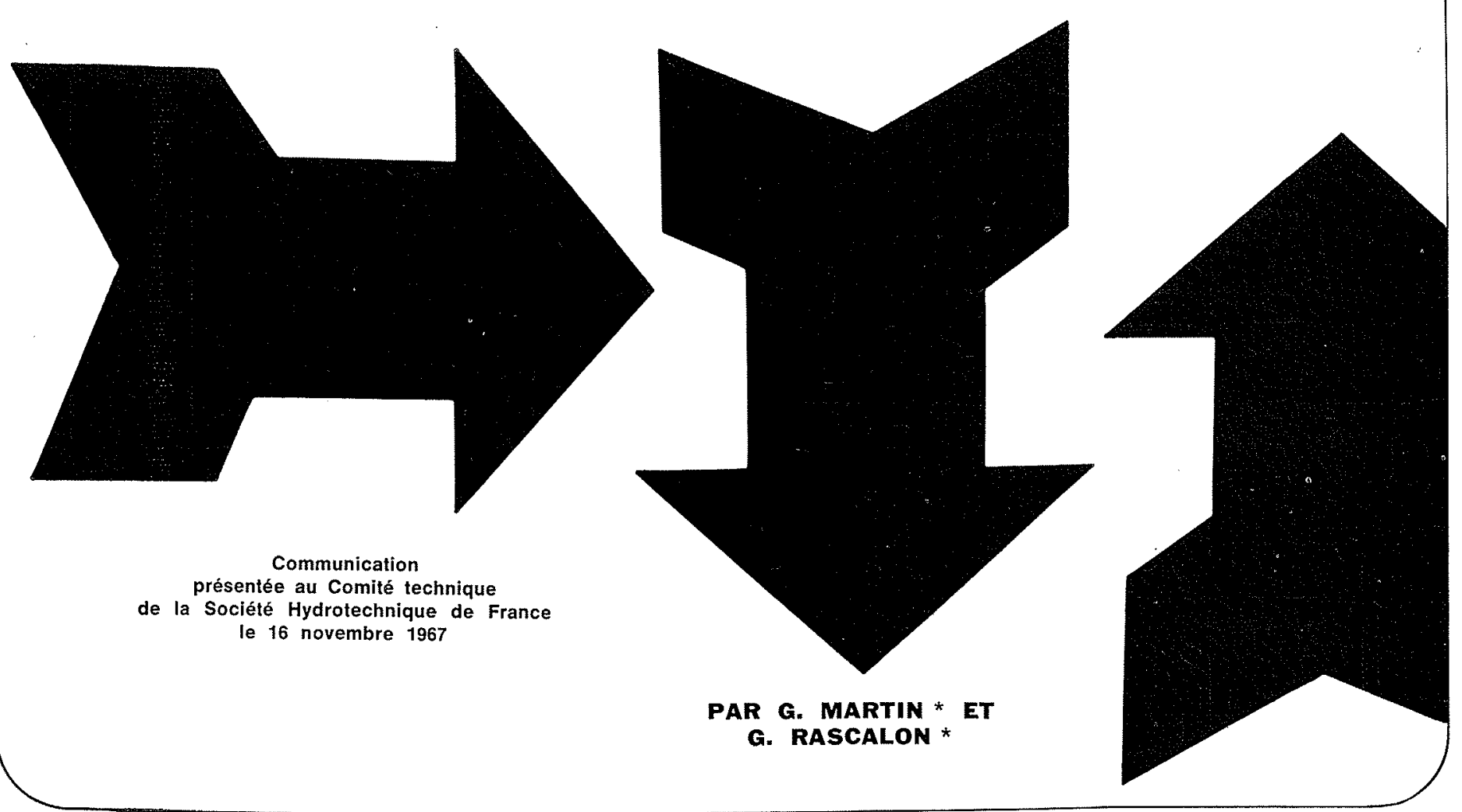

\section{Introduction}

But et caractères généraux.

Dans une turbine Kaplan, Ia marche à vide est obtenue par une conjugaison des pales de la roue et du distributeur qui conduit à la vitesse synchrone avec un débit « perdu » minimal.

Avec les dispositions habituelles de régulation, on engendre, lors d'une disjonction, des intumescences dans les canaux d'amenée et de fuite, par suite de la coupure rapide d'une partie importante du débit.

On peut remédier aux inconvénients qui résultent de l'existence de ces intumescences par l'utilisation d'un déchargeur, conjugué avec le vannage de la turbine.

Les investissements élevés nécessités par l'installation des déchargeurs classiques ont conduit à l'idée d'utiliser dans les installations de basses chutes les turbines elles-mêmes pour restituer des débits importants de marche à vide.

Dans un premier stade, on a exploité le procédé qui consiste à « déconjuguer 》 les aubages du dis-. tributeur et les pales du rotor.

On doit donc, dans ce cas, dissiper dans la machine une plus grande énergie. Les débits de marche à vide sont alors limités par les phénomènes

\footnotetext{
* Ingénieurs à la Société Grenobloise d'Etudes et d'Applica-
} tions Hydrauliques (SOGREAH), Grenoble. de cavitation el les instabilités qui résultent de la déconjugaison.

Dans ce type de fonctionnement, on a pu augmenter, d'une facon significative les débit déchargés en mettant en place me large aération du centre de la machine.

On a également développé un autre procédé qui consiste à combiner les positions des pales et du distributeur avec une ouverture partielle de la vanne de garde aval de la machine.

En fait, dans certaines réalisations industrielles, les deux procédés sont utilisés concurremment: Te premier pour les phases transitoires, le deuxième pour les régimes de longue durée.

Nous allons résumer les différents essais sur modèle réalisés dans nos laboratoires pour ces deux procédés et les comparer aux essais industriels.

Marche

en déchargeur déconjuguée Cas des Kaplan classiques

\section{Essais sur modèle réduit.}

Bien que ne permettant pas de représenter exactement et de mesurer les phénomènes de vibrations engendrés par les fonctionnements fortement déconjugués de la marche en déchargeur, les essais sur modèle ont permis de déterminer les fonctionnements qui semblaient les moins dangereux. 


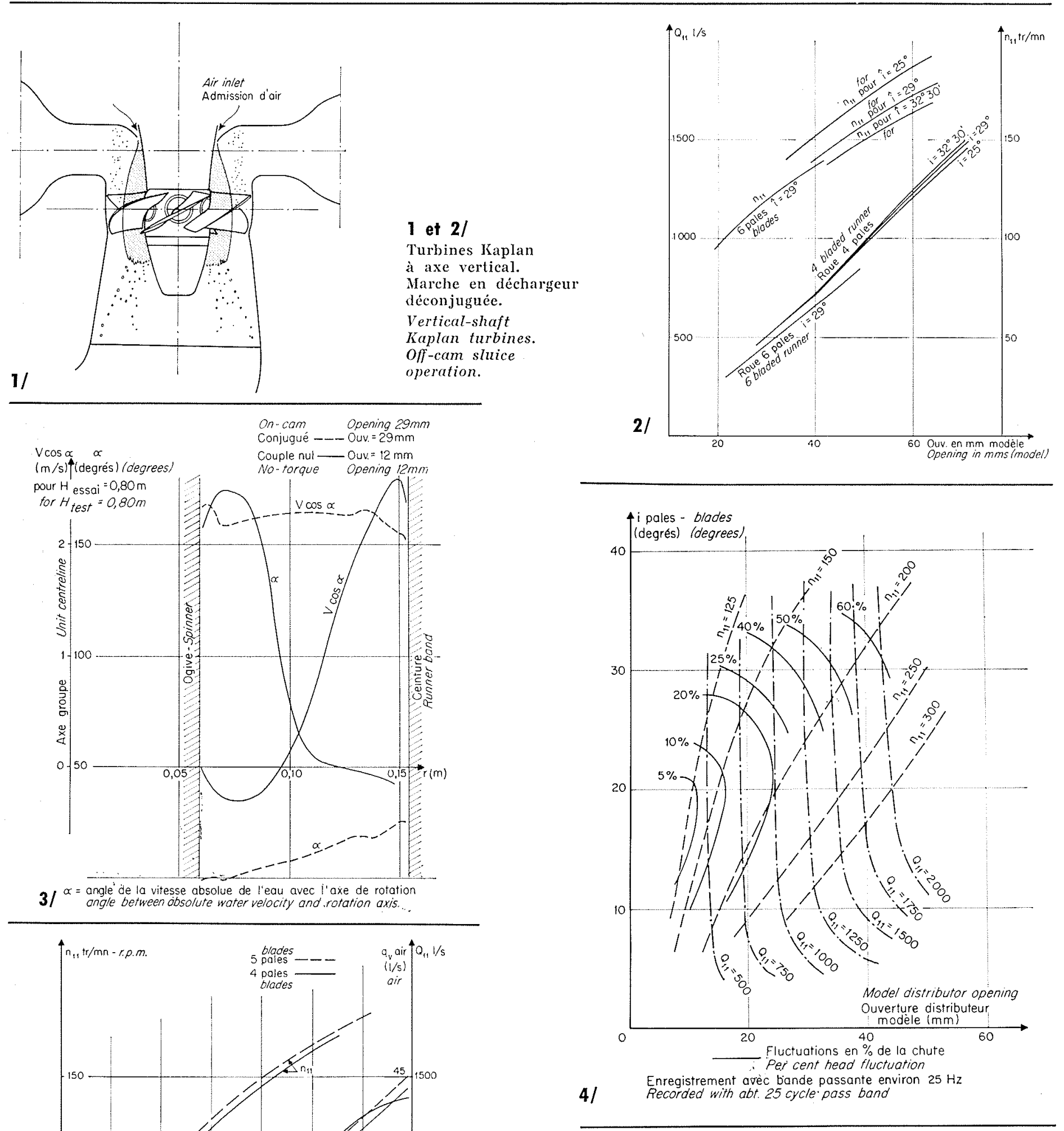

3/ Turbines Kaplan à axe vertical. Différence d'écoulement sous la rove en marche normale et marche en déchargeur. $i=29^{\prime \prime}$.

Vertical-shaft Kaplan turbines. Difference between flows underneath the runner in normal and sluice operation. $i=29^{\circ}$.

4/ Turbines Kaplan à axe vertical. Marche en déchargeur Vertical-shaft Kaplan turbines. Sluice operation.

5/ Turbines Kaplan à axe vertical. Marche en déchargeu déconjuguée. $\mathrm{i}=29^{\circ}$.

Vertical-shaft Kaplan turbines. Off-cam sluice operation. $i=29^{\circ}$. Model tests. 


\section{A - Caractéristiques générales.}

Les essais sur modèle ont été, pour la plupart, réalisés en similitude de Froude et de Thoma avec une machine industrielle.

Le premier objectif a été de choisir une vitesse de rotation de la machine voisine de la vitesse synchrone.

Lors d'une marche en déchargeur, la roue, ne fournissant qu'une puissance très faible, fonctionne comme un moulinet n'absorbant qu'une très faible partie de la chute, il en résulte que :

- le débit ne dépend pratiquement que de l'ouverture du distributeur;

- la vitesse dépend de l'ouverture du distributeur et de l'inclinaison des pales.

Les premiers essais réalisés avec une ouverture du distributeur relativement faible, ont montré :

- un anneau d'eau périphérique dans la roue (fig. 1) ;

- une zone centrale fortement perturbée, rendue apparente par un dégazage de l'eau.

On a également constaté que pour un même débit, le fonctionnement est moins perturbé lorsque les inclinaisons des pales de la roue sont grandes. On verra par la suite que c'est aux fortes inclinaisons que la dépression centrale est la plus grande.

Pour des vitesses voisines de la vitesse synchrone industrielle (fig. 2), pour les grandes inclinaisons des pales et une même ouverture du distributeur, les débits sont pratiquement indépendants du lype de pale, du nombre de pales et de la dimension du moyeu.

B - COMparaison ENTRE la MARCHE NORMALE E'T IA MARCHE EN DÉCHARGEUR.

\section{a) Essais sonde:}

Ces essais ont élé réalisés à un niveau de pression notablement plus élevé que celui qui correspondrait à la condition de Thoma.

Les diflérences d'écoulement sous la roue en marche normale et en marche en déchargeur apparaissent sur la figure 3 .

Les essais à la sonde permettent de déterminer d'une manière précise la zone annulaire dans laquelle se fait l'écoulement sain.

b) Essais en similitude de cavitation :

Pour une machine industrielle type, des essais ont été réalisés en similitude de cavitation (Froude et Thoma).

Les mesures de fluctuations de pression dans l'aspirateur montrent que les fluctuations sont une fonction croissante de l'inclinaison des pales et de l'ouverture du distributeur (fig. 4).

Les essais réalisés sans introduction d'air ont fait apparaitre que les aspects de cavitation sont différents de ceux observés en marche normale conjuguée :

- en marche en déchargeur, on observe très peu de cavitation en «poches accrochées » rappelant les cavitations en charge. Les cavités apparaissent d'abord dans la roue et se développent vers l'aval. En plus apparaît sous la roue une torche dissymétrique fluctuante, les phénomè- nes sont nettement plus violents qu'en marche normale avec apparition de claquements secs de cavitation;

-.- on peut en conclure que pour un régime normal, la cavitation, si elle existe, n'a pour effet que d'engendrer une érosion progressive des aubages; par contre, pour une marche en déchargeur, celte cavitation, de forme différente, est susceptible de provoquer des pulsations de pressions et des vibrations ayant une intensité telle qu'elles peuvent mettre en danger la machine, même si la durée d'un tel fonctionnement est limitée.

\section{C - Influence de L'Admission d'Air.}

Les essais ont été réalisés en similitude de cavitation.

La violence des phénomènes constatés et la localisation d'une dépression centrale nous ont conduit à essayer de reporter la dissipation d'énergie, à l'aval du rotor, dans la partie supérieure de l'aspirateur.

Une admission d'air a été réalisée à l'amont de la roue.

Le but était de rechercher les possibilités d'admission d'air atmosphérique.

On a constaté :

-- que pour un même débit déchargé, le débit d'air absorbé croît avec l'inclinaison des pales;

- que pour la même inclinaison des pales, lorsque la vitesse du groupe augmente, le débit d'air absorbé se sature, alors que le débit d'eau crôit régulièrement (fig. 5). Le débil d'air dont il est question ici est mesuré à la pression d'alimentation qui, sur le modèle, simule, en similitude de Froude-Thoma, la pression atmosphérique. Ce débit d'air est ramené, comme le débit d'eau, à un diamètre de roue de $1 \mathrm{~m}$ et une chute de $1 \mathrm{~m}$;

- qu'une poche centrale pleine d'air et un anneau liquide périphérique se maintiennent de façon stable, la dissipation d'énergie étant reportée à l'aval du rotor (fig. 1);

- que pour un même débit déchargé, les bruits d'ensemble de la turbine sont sensiblement réduits;

- que ces conditions d'écoulement stable et relativement moins bruyant ne peuvent être obtenues, pour une inclinaison donnée, que si l'ouverture du distributeur est maintenue inférieure à une certaine valeur. Si l'on ouvre le distributeur au-delà de cette valeur, la vitesse de rotation et le débit augmentent, les bruits et les claquements réapparaissent et l'admission d'air diminue. La poche d'air centrale se réduit, devient instable et se referme dans la roue; la dissipation d'énergie se fait alors partiellement à nouveau dans le rotor.

II est évident, puisqu’il s'agit d'air atmosphérique, que le débit d'air absorbé est fonction de la position du niveau aval. Il semble que le fonctionnement en déchargeur sera pratiquement correct tant que la poche d'air centrale permettra de reporter la dissipation d'énergie à l'aval des aubages de la roue. 


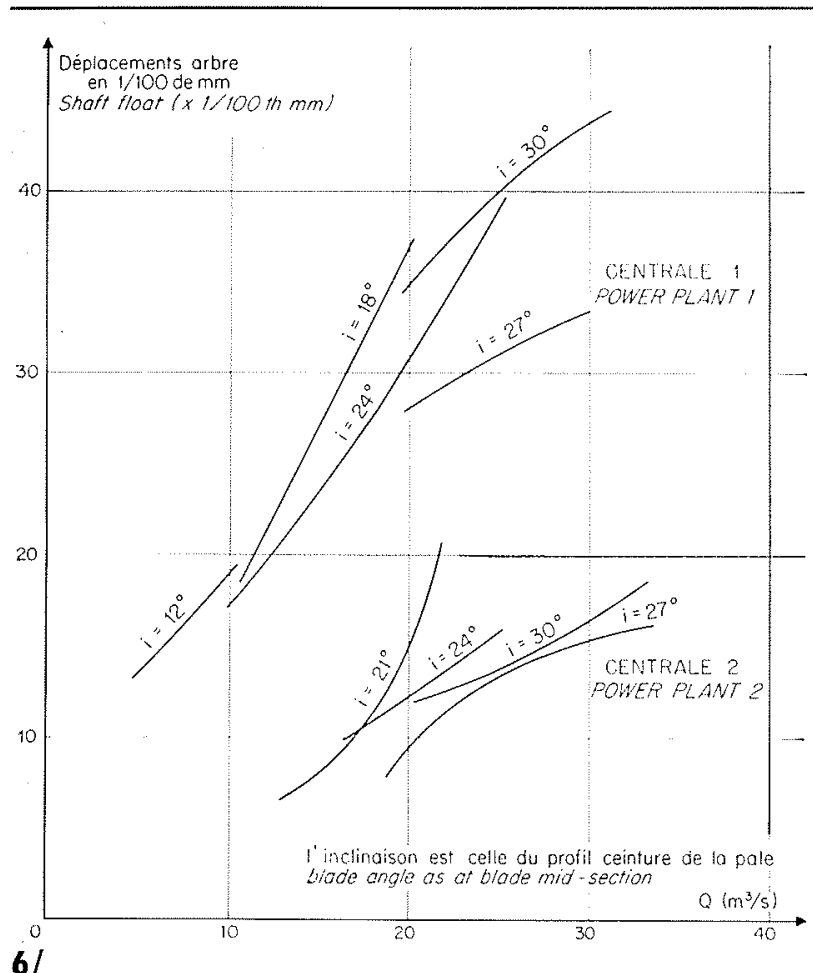

\section{6 et $7 /$}

Turbines Kaplan a axe vertical.

Marche en déchargeur déconjuguée.

Essais industriels.

Vertical-shaft

Kaplan turbines.

Off-cam

sluice operation.

Industrial tests.
$7 /$

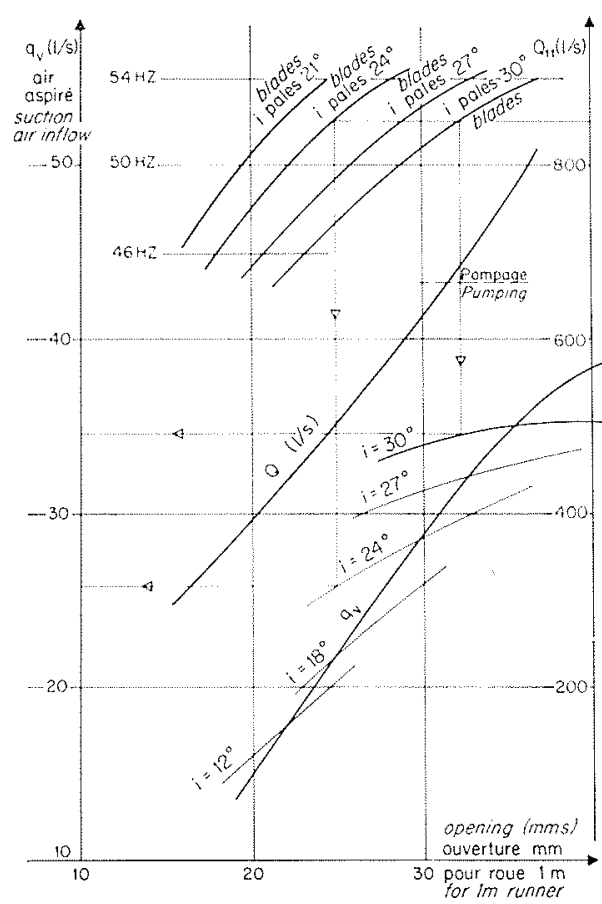

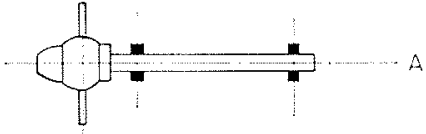
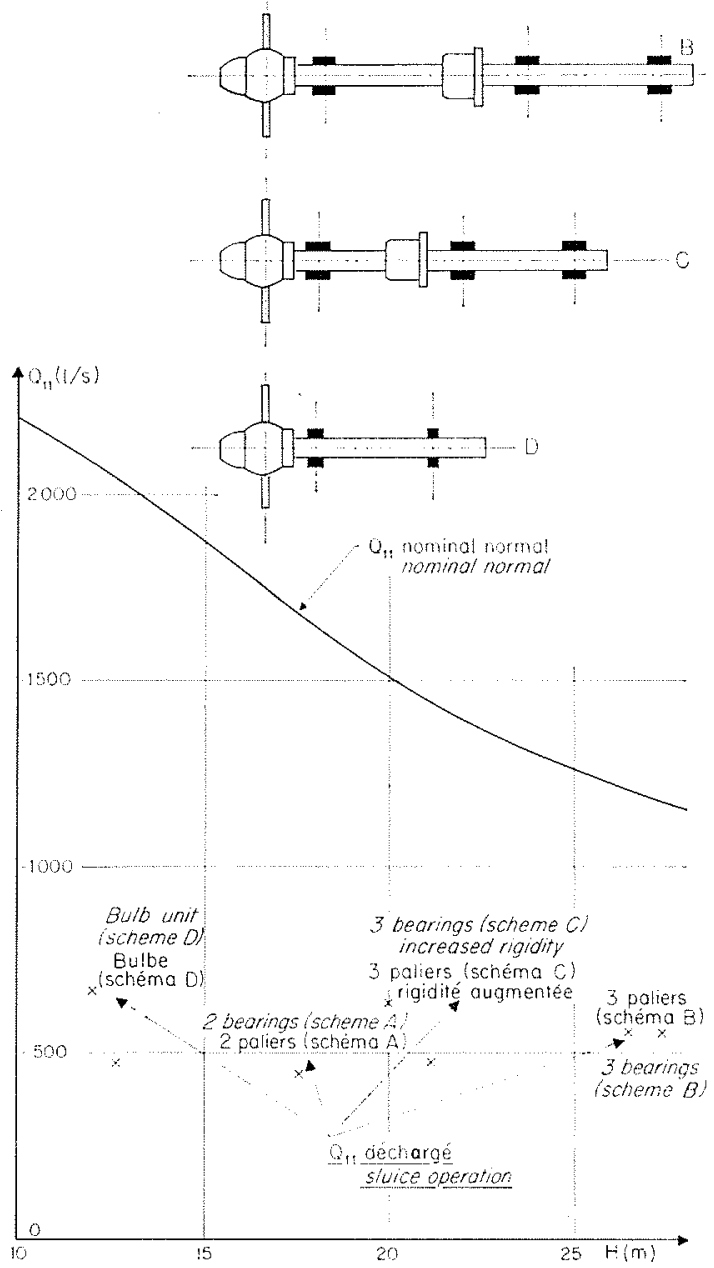

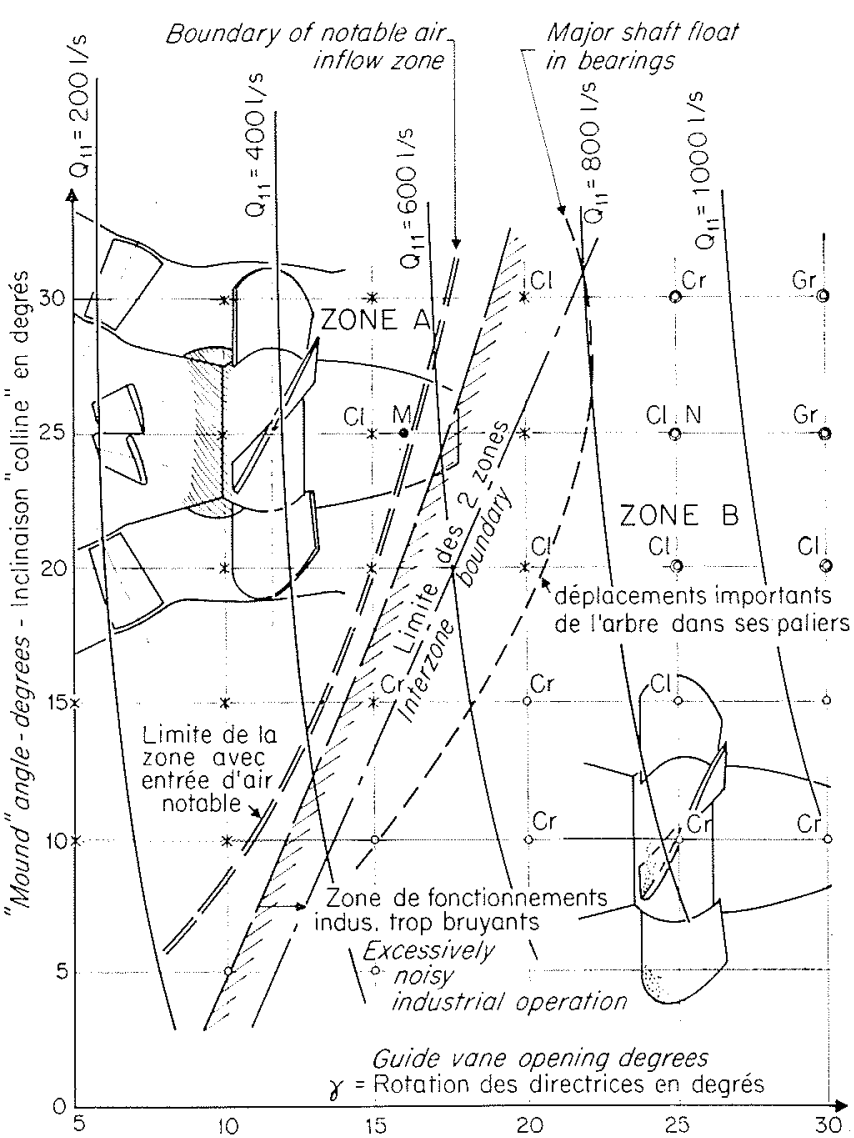

9/Groupe bulbe de rivière. Marche en déchargeur déconjuguée.

River bulb unit. Off-cam sluice operation.

8/ Turbines Kaplan classiques. Marche en déchargeur déconjuguée.

Conventional Kaplan turbines. Off-cam sluice operation. 


\section{Essais industriels.}

L'analyse des essais industriels a permis de définir d'une manière un peu plus précise les possibilités en marche en déchargeur des turbines Kaplan ainsi que les limites qui peuvent être obtenues par :

-une admission d'air;

- une augmentation de la rigidité des groupes.

Sur les machines industrielles, il est possible de mesurer des déplacements d'arbre, des efforts sur les paliers, des fluctuations de pression à l'amont ou à l'aval de la roue des débits d'air absorbé, les débits déchargés, etc.

Nous ne rapporterons ici que quelques unes de ces mesures.

\section{A - Anatyse succincte des essais.}

La figure 6, relative à deux turbines industrielles, montre que pour chaque inclinaison des pales les déplacements d'arbre sont une fonction croissante du débit déchargé.

On constate que les fonctionnements les meilleurs sont obtenus pour une forte inclinaison des pales pour un même débit déchargé (voisine de $27^{\circ}$ au profit ceinture).

Sur la figure 7 , on voit que, à débit déchargé constant, c'est avec les pales aux plus grandes ouvertures que le débit d'air absorbé est le plus grand.

On a également constaté que lorsque, pour une même inclinaison des pales, on augmente la vitesse de rotation, donc le débit déchargé, le débit d'air se sature et le groupe « pompe ».

Enfin, des essais comparatifs ont montré que pour les mêmes risques (poussées, perturbations, vibrations, déplacement d'arbre) les débits d'eau déchargés avec introduction d'air sont de 20 à $30 \%$ supérieurs à ceux obtenus sans introduction d'air.

\section{B - Comparaison des résultats obtrenus.}

L'observation des bruits, vibrations et déplacement d'arbre permet de juger dans chaque cas la valeur maximale de débit qui peut être obtenue en marche en déchargeur déconjuguée. Pour des turbines implantées et construites sans considération spéciale de ce type de fonctionnement, les valeurs constatées correspondent à un débit spécifique $Q_{11}$ voisin de $500 \mathrm{l} / \mathrm{s}$ et ceci quelle que soit la vitesse spécifique de la turbine (bien qu'apparemment subjectives, ces observations n'ont pas fait apparaitre d'équations personnelles).

Par augmentation de la rigidité, il est possible d'augmenter sensiblement ce $Q_{11}$, comme le montrent la figure 8 et les schémas.

Sur ce même graphique a été portée une courbe représentant la valeur moyenne des débits nominaux des groupes Kaplan classiques en fonction de la chute.

On voit que l'on peut par ce procédé de marche en déchargeur déconjuguée, décharger une fraction plus importante du débit nominal pour les groupes de fortes chutes que pour les groupes de basses chutes et qu'il faut pour ceux-ci chercher un autre procédé.

Il est évidemment possible de surdimensionner

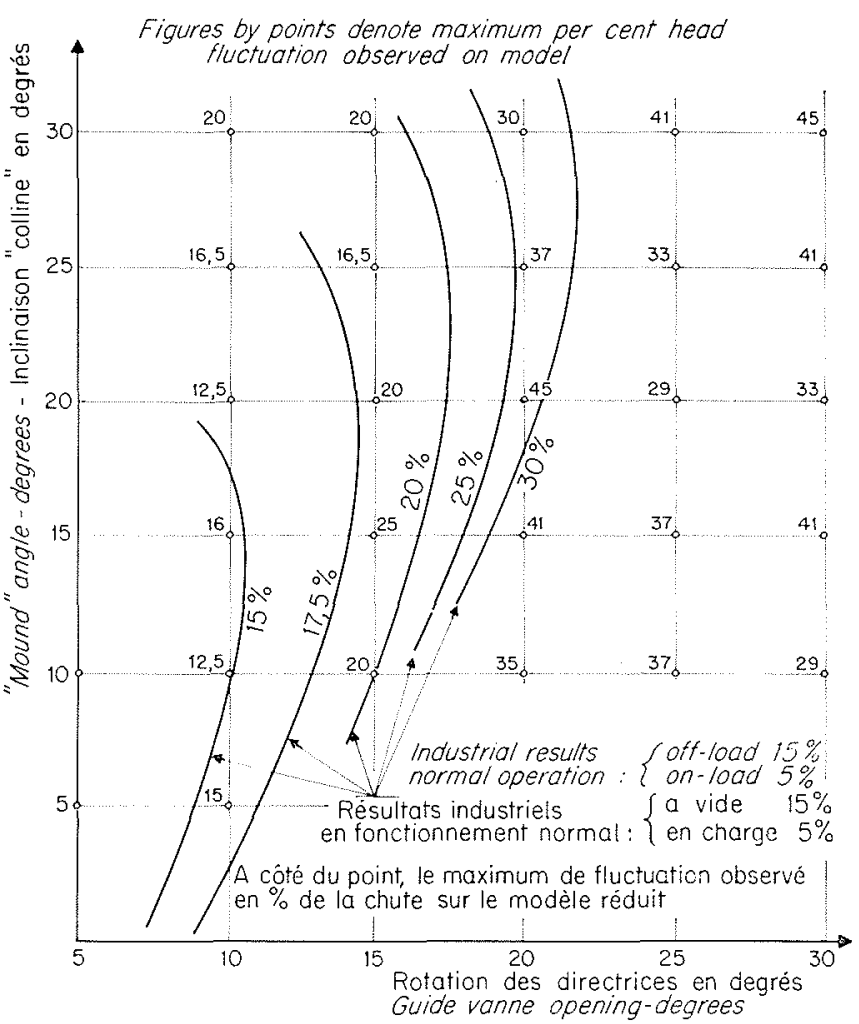

10/ Groupe bulbe de rivière. Marche en déchargeur déconjuguée. Fluctuation maximale au capteur aval, en $\%$ de la chute.

River bulb unit. Off-cam slatee operation. Maximum fuctuation at the domnstream pick-up (per cent of head).

les groupes pour augmenter le rapport entre le débit déchargé et le débit nominal. Cette méthode a l'avantage de permettre l'abaissement du niveau aval, donc une meilleure admission d'air et une augmentation du débit déchargé en même temps qu’elle réduit le $Q_{11}$ nominal. Ce procédé conduit à une augmentation du coût des machines; une étude économique peut permettre d'optimaliser et de juger de la rentabilité du projet.

\section{Marche en déchargeur déconjuguée Cas d'un groupe bulbe}

\section{Essais sur modèle réduit.}

Les essais sur modèle réduit ont été effectliés avec des coefficients de cavitation identiques à ceux de Ia machine industrielle.

Ils ont permis de définir dans un plan inclinaison-ouverture (fig. 9) deux zones où les fonctionnements sont de types nettement différents :

a) dans la zone $A$, on observe un décollement à l'amont des pales. Aucune trace de cavitation n'apparaît dans le rotor. Le fonctionnement est sans bruit notable, la zone de dépression est favorable à une entrée d'air atmosphérique;

b) dans la zone $\mathrm{B}$, le décollement amont a disparu, la cavitation apparaît à l'entrée des aubages au voisinage de la ceinture. Le fonctionnement est 
bruyant avec claquements liés à des perturbations importantes de l'écoulement. A priori, il sera impossible de fonctionner de manière continue en déchargeur dans cette zone.

Les fluctuations de pression augmentent avec I'ouverture du distributeur mais sont pratiquement indépendantes de l'inclinaison des pales (fig. 10 et 11).

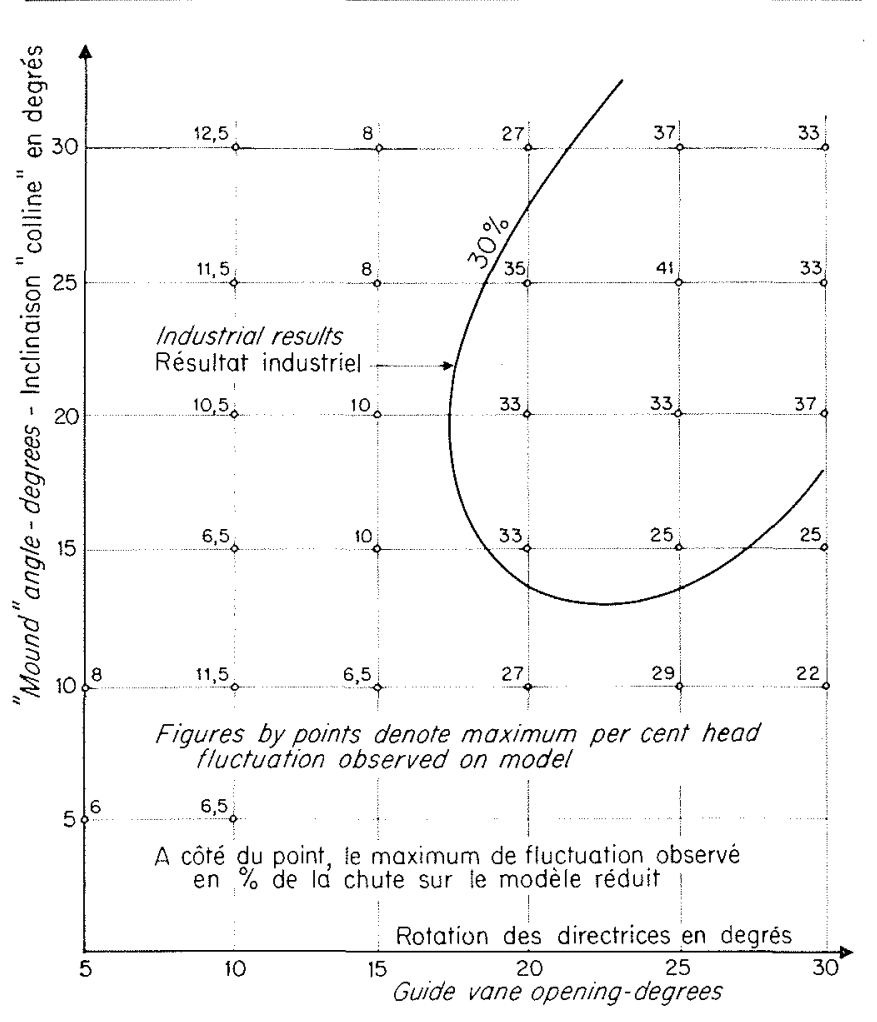

11/ Marche en déchargeur. Essais sur modèle réduit de bulbe de rivière. Fluctuation maximale au capteur amont, en $\%$ de la chute.

Sluice operation. River bulb unit model tests, Maximum fluctuation at the upstream pick-up (per cent of head).
Les figures 12 et 13 sont relatives aux points de fonctionnements :

- M dans la zone $A$

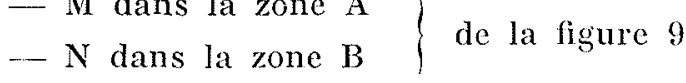

Les essais sur modèle des groupes bulbes ont été identiques à ceux réalisés sur les groupes Kaplan classiques. Ils montrent une bomne analogie des phénomènes hydrauliques pour les deux types de machine lors des fonctionnements en déchargeur déconjugués.

\section{Essais industriels.}

Industriellement, les essais ont été effectués à inclinaison constante. Le distributeur était ouvert progressivement pour augmenter le débit déchargé.

Pour chaque point stabilisé, on a mesuré ou enregistré : les débits d'air absorbé par la turbine, les pressions et fluctuations de pression en différents points du conduit hydraulique, les déplacements d'arbre. Des observations ont été notées sur les bruits et le comportement général de la machine.

On a ainsi déterminé une limite d'utilisation représentée, en fonction de l'inclinaison des pales et de l'ouverture du distributeur, sur la figure 9 . Cette limite est parallèle et légèrement inférieure en débit à celle définie sur modèle réduit et séparant les deux zones $\mathrm{A}$ et $\mathrm{B}$.

Sur le graphique 14 on remarque que, pour une même inclinaison des pales, lorsque l'ouverture du distributeur, donc le débit, et la vitesse du groupe augmentent, le débit d'air absorbé se sature brutalement. La limite du fonctionnement possible industriel en déchargeur correspond pratiquement à cette saturation brutale du débit d'air absorbé.

L'examen montre que, comme pour les Kaplan classiques, les grandes inclinaisons des pales sont les plus favorables pour passer les plus grands débits possibles en marche en déchargeur.
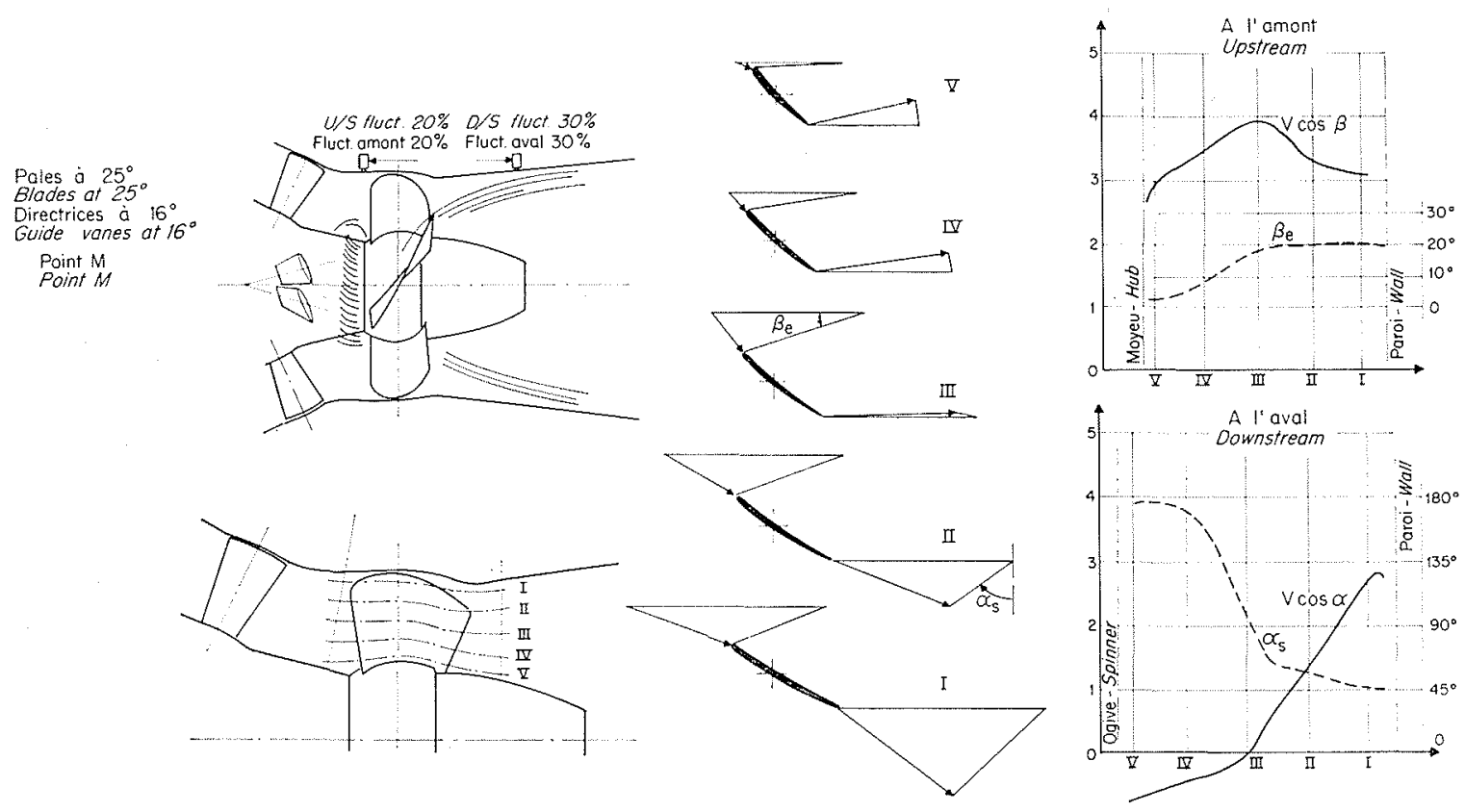

12/ Narche en déchargeur déconjuguée. Bulbe iviere. off-cam sluice operation. River bulb unit. 
13/ Marche en déchargeur déconjuguée. Bulbe rivière. operation.

River bulb unit. Off-cam sluice

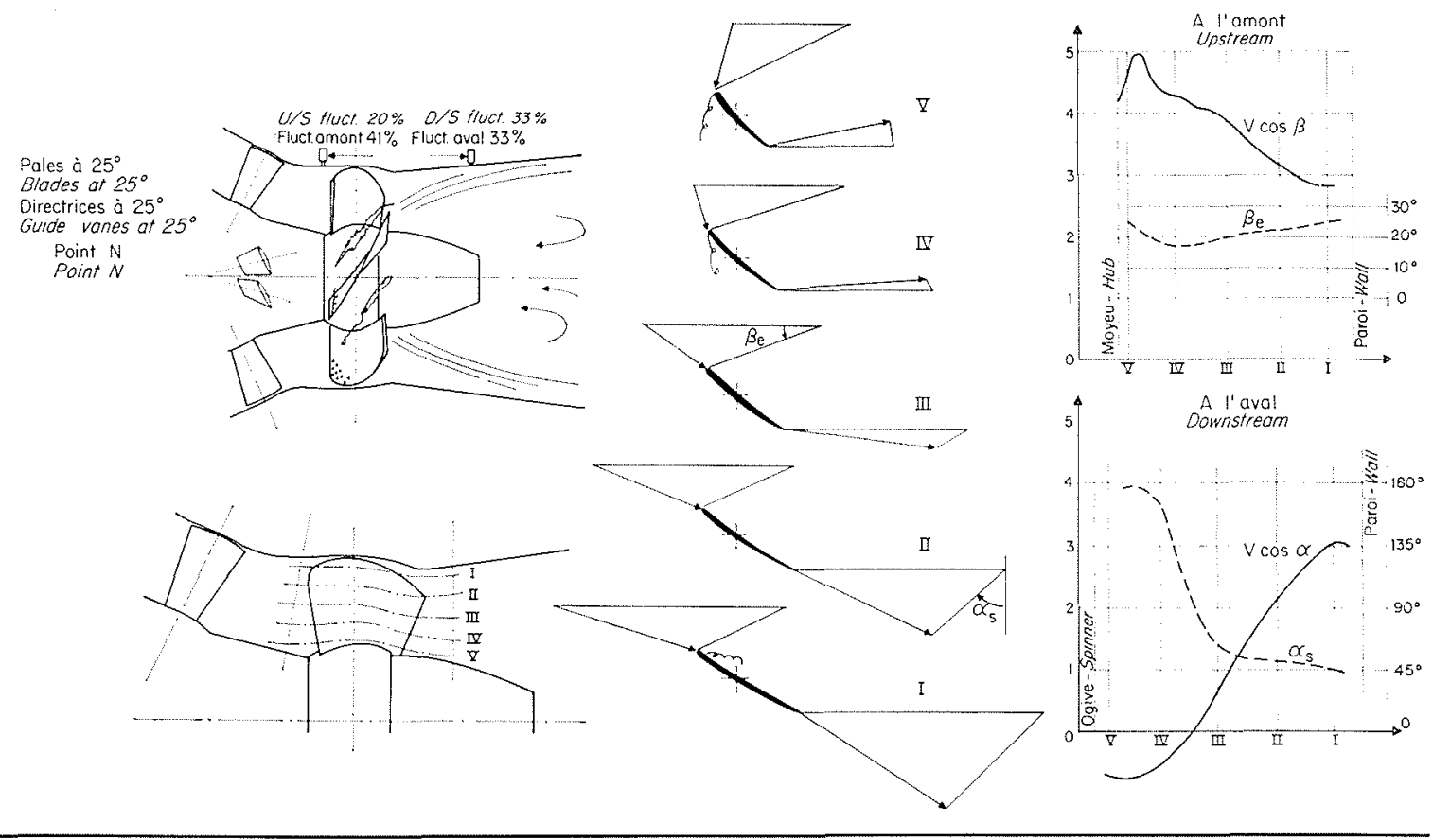

Sur la figure 8 est porté le point représentatif relatif à ce groupe bulbe (repère $D$ ); sa conception « ramassée » schéma $D$ lui donne une rigidité relative plus importante que celle d'un groupe classique et permet d'atteindre un $Q_{11}$ déchargé de $680 \mathrm{l} / \mathrm{s}$.

\section{Marche en déchargeur avec vanne aval d'un groupe bulbe}

\section{Essais sur modèle réduit.}

L'idée directrice est de reporter la dissipation d'énergie à l'aval du groupe. La présence de la vanne aval partiellement fermée permet à la roue de la turbine de fonctionner comme un moulinet sous faible chute avec forte contrepression. Ceci permet de fonctionner à pleine ouverture du distributeur et des pales dans des conditions relativement favorables d'incidence sur les profils du rotor.

Les essais sur modèle ont été réalisés en similitude de Froude-Thoma avec une machine industrielle.

Tous les essais ont élé réalisés avec le distributeur ouvert au maximum.

Les résultats relatifs à des inclinaisons du profil ceinture de 25 et $33^{\circ}$ sont portés sur les figures 15 et 16.

Sur le réseau de courbes, débit en fonction de l'ouverture de la vanne aval pour différentes chutes, ont été définies deux limites caractéristiques :

a) apparition des premières bulles de cavitation sur les aubages. Lorsque apparaissent les premières bulles, on ne discerne aucun bruit audible sur le modèle;

b) cavitation peu développée, sensiblement identique visuellement, en étendue sur le profil, à celle admise dans un fonctionnement en charge. Cette cavitation apparait sur l'intrados du pro-

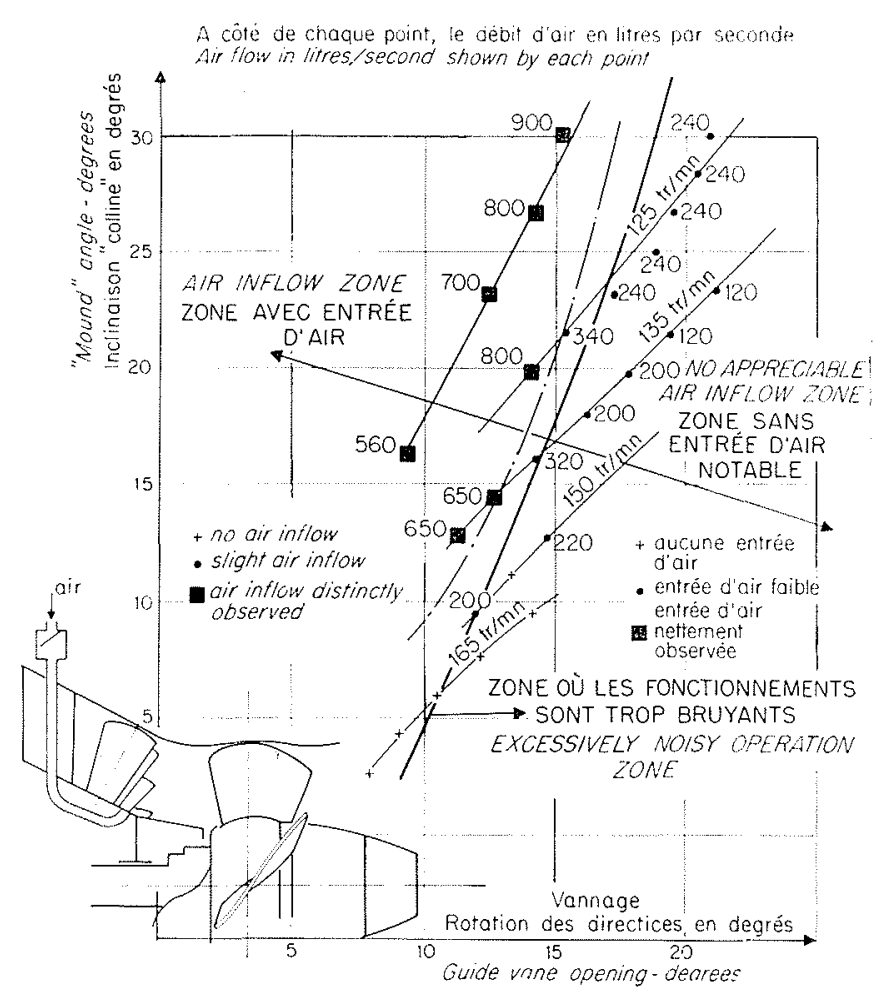

14/ Groupe bulbe de rivière. Marche en déchargeur déconjuguée. Entrée naturelle de l'air par les clapets sur la machine industrielle.

River bulb unit. Off-cam sluice operation. Natural air entry through the inlet values on an industrial machine.

fil, alors qu'elle apparaît sur l'extrados lors d'une marche normale; elle n'a pas non plus l'aspect de poche lisse de ce type de fonctionnement.

Cette cavitation d'intrados est relativement bruyante avec apparition de crépitements.

La proximité de la vanne aval, située à environ 


\section{G. MARTIN et G. RASCALON}

deux fois le diamètre de la roue à l'aval de celle-ci, pouvail faire craindre un écoulement dissymétrique dans la roue lorsque l'ouverture de la vanne ćtait faible, c'est-à-dire pour les fortes chutes.

La figure 17 montre qu'il n'en est rien et que pour un niveau amont constant, l'apparition des premières bulles de cavitation est indépendante de la position de la vanne, donc de la chute. On constate également que, pour un même risque, le débit déchargé le plus grand est obtenu avec la plus grande inclinaison des pales.

\section{Essai industriel et comparaison avec le modèle réduit.}

Les essais industriels montrent que les limites acceptables sur le prototype correspondent pratiquement sur le modèle réduit à l'apparition des premieres bulles de cavitation sur les pales (fig. 15 ct 16 ).

L'apparition de la cavitation, de vibrations et de chocs apparait d'une manière assez brutale pour une augmentation faible du débit.

Le sigma critique de cavitation en déchargeur, rapporté à la chute vraie sur la roue, est environ six fois plus grand que celui correspondant à la même inclinaison et à la même ouverture sous une chute normale de fonctionnement. Ceci s'explique par une mauvaise incidence sur l'arête d'entrée des pales de la roue lors d'un fonctionnement à l'emballement.

La figure 18 montre les possibilités de décharge d'un groupe équipé avec vanne aval. Il est vraisemblablement encore possible d'augmenter le débit déchargé par une surouverture du distributeur. Sur cette même figure, nous avons fait figurer une courbe qui représente le débit qui pourrait être restitué par marche en déchargeur conjuguée. Pour calculer cette dernière courbe, nous nous sommes basés sur un $Q_{11}$ d'environ $650 \mathrm{l} / \mathrm{s}$.

\section{Conclusion}

Les analyses sur modèle réduit et les essais industriels ont montré qu'on pouvait, par déconjugaison, augmenter substantiellement le débit de marche à vide d'une turbine de type Kaplan. La valeur des débits admissibles peut être augmentée par une large admission d'air au centre de la machine et par une conception plus rigide du groupe. De toute façon, le débit restitué est au plus de
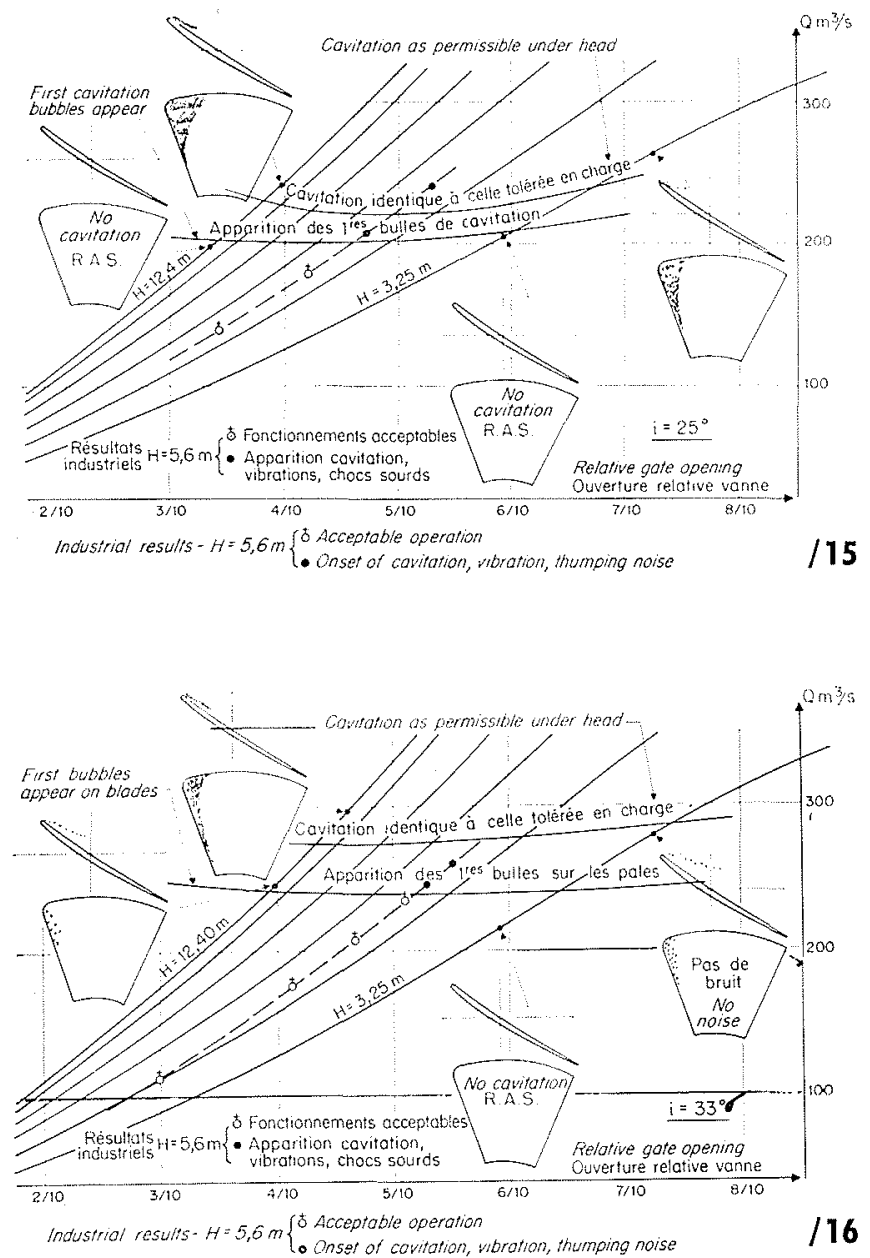

15 ef 16/Groupe bulbe de rivière. Marche en déchargeur avec vamne aval / River bulb unit. Sluice operation with a gate dounstream.

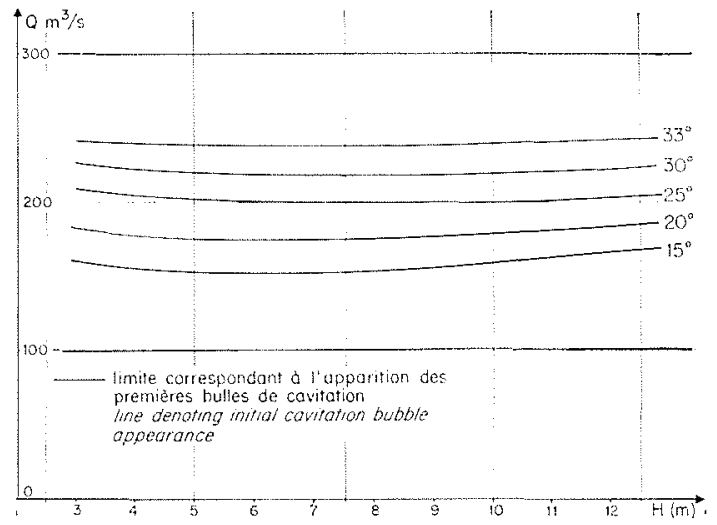

17/ Groupe bulbe de rivière. Possibilité de décharge avec vanne aval / Riper bulb unit. Relief capacity with a gate downstream.

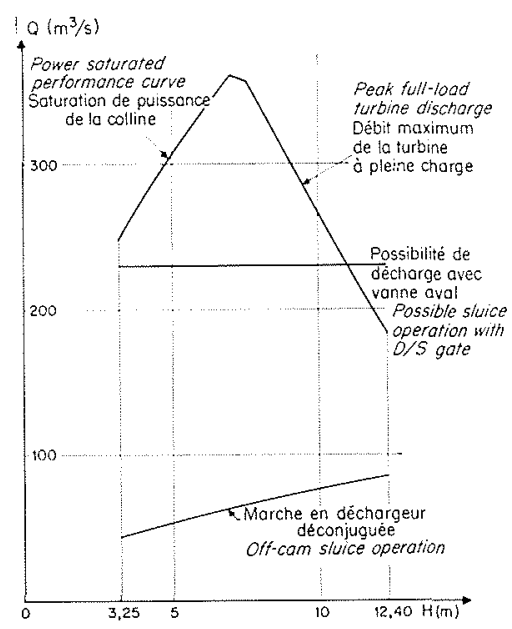

18/ Groupe buibe de rivière. Marche en déchargeur avec vanne aval / River bulb unit. Slnice operation with a gate downstream. 
l'ordre de $30 \%$ du débit nominal pour les machines de basse chute et peut atteindre $50 \%$ dans les cas les plus favorables dans les chutes de l'ordre de 20 a $25 \mathrm{~m}$. Pour les machines à grande puissance spécifique, donc à calage profond, l'aération naturelle devient difficile ou impossible et les débits restitués peuvent s'en trouver diminués.

Le procédé de marche en déchargeur par ferme- ture partielle de la vanne aval permet d'atteindre les débits restitués notablement plus importants et ceci même pour des durées de fonctionnement prolongées. Toutefois, la manouvre de la vanne étant en général sensiblement plus lente que celle des organes normaux de régulation, il est souvent intéressant d'utiliser transitoirement la déconjugaison de façon à réduire les écarts de débit.
M. le Plésident remercie M. Manew pour son exposé sur les importants travaux effectués par SOGREAH dans le domaine visé

M. RÉmÉviéras rappelle que, dans quelques cas, on a pu réduire très sensiblement les vibrations et bruits associés a certains régimes hydrauliques perturbés qui prennent naissance dans certaines turbines et leurs aspirateurs, en disposant dans ces derniers un systeme défolmable analogue, en principe, à celui qu'il a présenté ici pour réduire l'amplitude des coups de bélier $\left(^{*}\right)$ dits de « felmeture rapide $\gg$. Un mode de realisation industriel ingénieux consiste à aménager un réservoir d'air (en communication avec la partie inf'érieure de l'écoulement) dans la cloison médiane creuse qui existe dans certains aspirateurs de turbine. Un autre procédé consisterait à améliorer (en s'inspirant de ce qui a été fait pour les compresseurs d'air hydrauliques), la qualité de l'émulsion de bulles d'air dans l'eau provoquée par les injections d'air utilisées sur certaines turbines pour combattre la cavitation.

M. Martin ne pense pas que de tels procédes soient ici efflcaces, car l'origine des phénomènes se trouve dans la roue ou à l'aval et dans son voisinage immediat.

M. Campuas se réfère a la figure 4 présentée par M. ManTh et note que l'amplitude des fuctuations dans l'aspirateur en vamnage bloque est une fonction croissante de l'ouverture des pales. Or, le plus souvent, le régime est dans l'ensemble plus stable à l'ouverture maximale de la rone. Cette anomalie n'est-elle pas due à l'existence d'une oscillation à basse fréquence $(0,5$ à $2 \mathrm{~Hz})$ qui se superposerant aux fluctuations rapides ef augmenterait l'amplitude entre cretes sans que le danger ponr la machine soit plus grand?

D'une manière générale, les amplitudes indiquées résultent-elles d'un même type d'oseillation ou de la superposition de deux phénomènes de caractères distincts (ce qui est fréquent daus l'aspirateur).

M. Martax répond que l'on a enregistré des fluctuations très lentes et que la figure présentée ne comporte pas assez de points pour donner une bonne représentation du phénomène.

(*) G. Rśóndéras. - Dispositif simple pour réduire la célérité des ondes élastiques dans les conduites en charge. La Houille Blanche, no $\mathrm{A} / 1952$
M. Campas remarque également que la saturation en débit d'air aspiré est conforme aux observations faites sur l'écoulement entre distributeur et roue. En raison de la diminution de la rotation de leau, on constate qu'a partr d'une certaine ouverture du distributeur, la pression près de l'axe tend à remonter malgré l'augmentation du débit décliargé.

Le choix des limites à ne pas dépasser dans la marche en déchargeul par déconjugaison des pales et du vannage est difficile, car une turbine est un ensemble trop complexe pour qu'on puisse utiliser de simples mesures d'extensométrie et un critère de résistance à la fatigue du métal. Il faut fairc intervenir les vibrations dont les effets a long terme sont quantitativement difficiles à prévoir. Dans certains cas, les limites admises par la D.T.G. (E.D.F.) paraissent un peu superieures à celles définies par M. Martin.

M. Ie President demande pourquoi, à Pierre-Bénite, les entrées d'air prévues initialement ont été supprimées ensuite.

M. Cazenave répond qu'avec le procédé utilisant une vanne aval rapide, il n'y a, au cours du régime transitoire, aucun moment où la pression en amont de la roue devient inférieure à la pression atmosphérique. Ce point a été vérifié par le calcul analogique et par l'expérience industrielle. Dès lors, les entrées d'air deviennent inutiles et constituent une petite complication (soupapes). On les a donc supprimées.

M. le Président pose encore deux questions à M. Cazenave : $1^{\circ}$ Quel est à Vallabrègues la valeur du débit maximal « en déchargeur » en pourcentage du débit nominal de la turbine?

$2^{\circ}$ Le groupe « bulbe $\gg$ a une ligne d'arbre qui parait mieux guidée que celle du groupe Kaplan classique. En résulte-t-il un meilleur fonctionnement en ce qui concerne l'importance des vibrations au cours des régimes perturbés tels que cenx qui peuvent survenir dans le fonctionnement en déchargeur?

A la première question, M. Cazenave répond que le débit « en déchargeur $»$ d'un groupe atteint $70 \%$ de son débit nominal. Pour l'ensemble de l'usine, compte tenu d'un groupe tombant en panne au moment même du déclenchement, on arrive à un débit « en déchargeur » égal à $58 \%$ du débit nominal.

La seconde question recevra une réponse au cours de l'exposé de M. Campmas. 